\title{
Nonlocal splitting of photons on a nonlinear chip
}

\author{
Frank Setzpfandt $1,2, *, * *$, Alexander S. Solntsev ${ }^{1, *}$, and Andrey A. Sukhorukov ${ }^{1}$ \\ ${ }^{1}$ Nonlinear Physics Centre, Research School of Physics and Engineering, Australian National University, Canberra, ACT 2601, Australia \\ ${ }^{2}$ Institute of Applied Physics, Abbe Center of Photonics, Friedrich-Schiller-Universität Jena, Max-Wien-Platz 1, 07743 Jena, Germany \\ * these authors contributed equally \\ ** corresponding author: f.setzpfandt@uni-jena.de
}

Compiled October 28, 2016

In spontaneous parametric down-conversion (SPDC), a pump photon spontaneously splits into signal and idler photons in media with quadratic nonlinearity. This phenomenon is the most widely utilized source of entangled photons with multiple applications in quantum information technology. SPDC on a chip is usually treated as a local process, meaning that signal and idler photons are created in the same position where the pump photon is destroyed. We reveal that this locality condition can be violated in an array of coupled waveguides. By utilizing higher-order modes of individual waveguides, it is possible to destroy a pump photon in one waveguide and generate signal and idler photons in other waveguides. This phenomenon of nonlocal photon-pair generation opens new opportunities for the engineering of spatial photon entanglement. (c) 2016 Optical Society of America

OCIS codes: (130.4310) Integrated optics, Nonlinear; (270.0270) Quantum optics; (130.3120) Integrated optics devices.

http://dx.doi.org/10.1364/ao.XX.XXXXXX

SPDC [1] is a popular process for the generation of entangled photon pairs, which are the fundamental ingredient for many quantum applications, including quantum-cryptography, -teleportation and -computer algorithms [2]. Quantum state engineering with SPDC has been experimentally realized using different degrees of freedom of photon pairs, e.g. polarization $[3,4]$ or spectral distribution $[5,6]$. The integration of photon-pair sources into waveguide circuits has recently become an important challenge for the realization of quantum applications [7]. Due to the discrete nature of integrated optical waveguides, providing many separated spatial modes, state engineering by control over the spatial degrees of freedom is a viable approach. The simplest examples demonstrate generation and control over two-photon quantum states in two waveguides [8-11]. In recent years nonlinear waveguide arrays (WGAs), regular arrangements of coupled optical waveguides as sketched in Fig. 1(a), have attracted interest as quantum state generators due to the large number of spatial output modes [12-18]. Nonlinear WGAs are able to generate tunable spatially entangled two-photon states, which has been recently shown experimentally [19].
Aside from cases of complex phase variation in bulk crystals [20-22], SPDC is usually local, meaning that a pump photon is destroyed in the same microscopic volume of space where a pair of photons is created. In all works in integrated optical platforms up to now this resulted in the pair of photons appearing in the same waveguide where the pump photon is destroyed. However, only the first modes at all frequencies were taken into account. For classical second-harmonic generation in WGAs it has been shown that interactions with higher order modes can be precisely controlled [23-25] and enable nonlinear coupling processes [26], i.e. parametric wavelength conversion into neighboring waveguides. In this contribution we analyze quantum state generation by SPDC with higher order modes in nonlinear WGAs and show that nonlinear coupling enables on-chip nonlocal splitting of one pump photon into signal and idler modes localized in different waveguides. Here, the photon pairs are generated in a microscopic volume between the waveguides, but deterministically populate certain combinations of overlapping modes in adjacent waveguides. This opens new ways for the engineering of the spatial properties of generated photon pairs.

We consider a one-dimensional WGA with period $d$ as sketched in Fig. 1(a) where the individual waveguides are homogeneous along the propagation direction $z$. Most realistic waveguides with second-order nonlinearity have a refractive index profile symmetric with respect to the transverse direction $y$. Hence, the guided modes can be classified as even (symmetric) or odd (anti-symmetric) with respect to reflection at the waveguide center as shown in Fig. 1(b). We define a symmetry parameter $S_{m}, m \in[\mathrm{p}, \mathrm{s}, \mathrm{i}]$ for the pump, signal, and idler modes, respectively, which is $S_{m}=1$ for even and $S_{m}=-1$ for odd modes. Both TE and TM modes in typical have only one non-negligible electric field component. Below we are considering TM modes with electric field linearly polarized along $x$. The electric field of the modes obeys $e_{m}(x, y)=S_{m} e_{m}(x,-y)$ with respect to the symmetry axis of the refractive index profile. During propagation, the occupation of the modes, i.e. the number of photons propagating in each spatial mode, changes by means of different coupling mechanisms. Evanescent overlap of the modes in adjacent waveguides leads to linear coupling, thus inducing a finite probability for photons to hop to the next waveguide described by the coefficients $c_{m}$, which are $c_{m} \geq 0$ for $S_{m}=1$ and $c_{m} \leq 0$ for $S_{m}=-1$ [27].

The quadratic nonlinearity facilitates SPDC [1]. Up to now this process was considered only locally, with pump, signal, and 


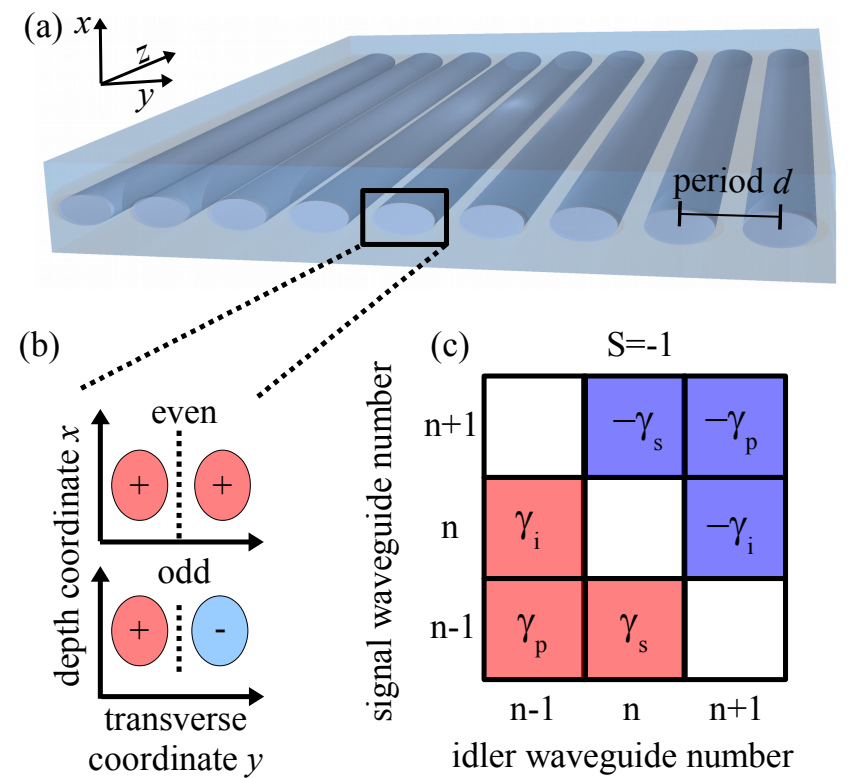

Fig. 1. (a) Sketch of a waveguide array. (b) Definition of even and odd waveguide modes. (c) Schematic representation of nonlinear interactions in the nonlocal case with $S=-1$.

idler photons in the same WGA waveguide. For classical SHG it was shown recently that evanescent overlap of modes leads to nonlinear interactions between neighboring waveguides [26]. In this nonlocal regime, at least one of the interacting photons resides in a neighboring waveguide. In SPDC, each of the three interacting photons could be shifted left or right, leading to different new interaction scenarios. The efficiencies of all nonlinear processes are determined by the nonlinear field overlap

$$
\gamma\left(n_{\mathrm{p}}, n_{\mathrm{s}}, n_{\mathrm{i}}\right)=\varepsilon_{0} /\left(2 \pi P_{0}\right) \iint \chi_{333}^{(2)} e_{n_{\mathrm{p}}}^{\mathrm{p}} e_{n_{\mathrm{s}}}^{\mathrm{s} *} e_{n_{\mathrm{i}}}^{\mathrm{i} *} \mathrm{~d} x \mathrm{~d} y,
$$

with the normalization power $P_{0}$, the nonlinear susceptibility $\chi_{333}^{(2)}$, and the waveguide numbers $n_{m}$ corresponding to a transverse coordinate. The local nonlinear coefficient is $\gamma_{l}=$ $\gamma(n, n, n)$ and the six possible nonlocal coefficients are $\gamma_{p \pm}=$ $\gamma(n \pm 1, n, n), \gamma_{s \pm}=\gamma(n, n \pm 1, n), \gamma_{i \pm}=\gamma(n, n, n \pm 1)$.

In a symmetric waveguide as considered here, the number of nonlocal coefficients can be reduced by defining $\gamma_{m}=\gamma_{m+}=$ $S \gamma_{m-}$, where the SPDC symmetry parameter of the interaction, $S=S_{\mathrm{p}} S_{\mathrm{s}} S_{\mathrm{i}}$, depends on the properties of the interacting modes. For $S=1$ the nonlocal nonlinearities are usually much smaller than the local ones, $\gamma_{m} \ll \gamma$, and can be neglected. However, for $S=-1$ local nonlinear interactions disappear due to the vanishing mode overlap $\left(\gamma_{l}=0\right)$ [26], and nonlocal SPDC becomes the dominant contribution. For this nonlocal case, all remaining interactions are shown in Fig. 1(c) with the corresponding nonlinear coefficients, where red (blue) denotes the positive (negative) sign of the coefficients. Here, signal and idler appear either in the same waveguide next to the pumped waveguide or are even directly generated in spatially separated modes.

Having established a mechanism for nonlocal generation of photon pairs, we now derive a general description of the two-photon state resulting from SPDC interactions in WGAs to analyze the effects of the nonlocality. The Hamiltonian describing the interactions in the WGA system, assuming narrow-band filtered degenerate signal and idler and the undepleted pump
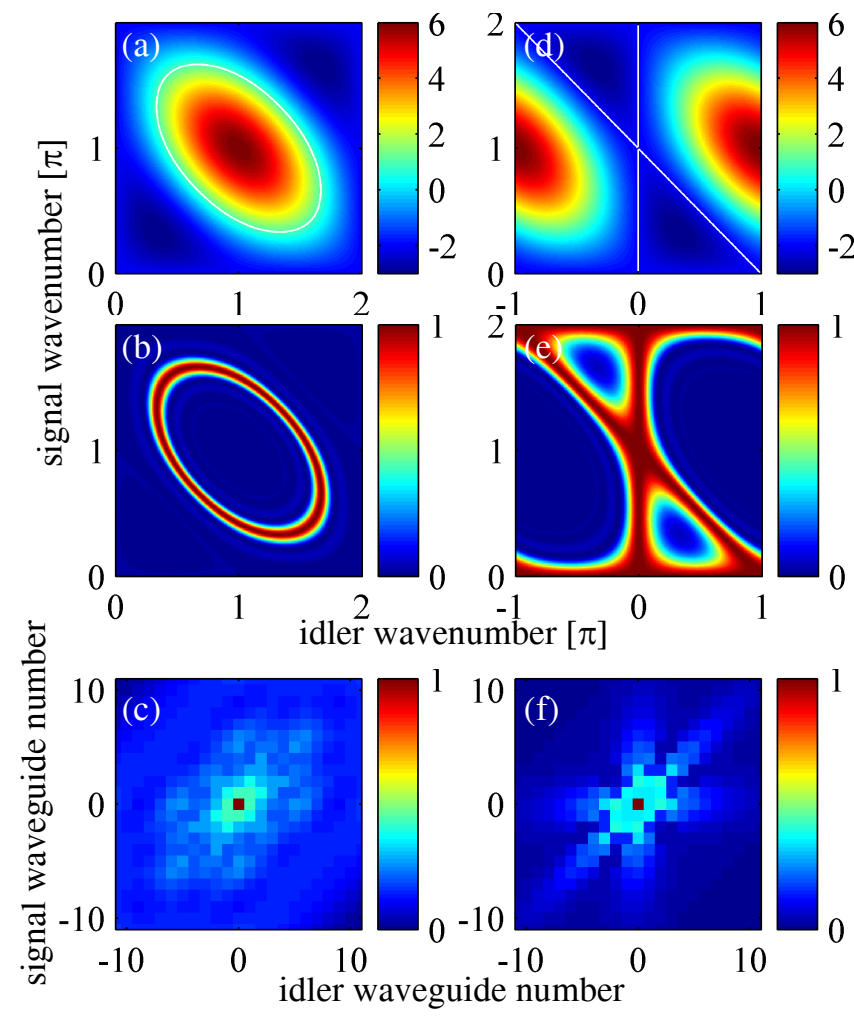

Fig. 2. Photon-pair generation with local nonlinear interaction and $S_{\mathrm{p}}=S_{\mathrm{s}}=S_{\mathrm{i}}=1$, (a-c) $\Delta \beta_{0}=-1$ and (d-f) $\Delta \beta_{0}=2$. In phase mismatch functions $(\mathrm{a}, \mathrm{d})$, the white lines indicate phase matching. The correlations in $k-(b, e)$ and real $(\mathrm{c}, \mathrm{f})$ space are shown for propagation length $L=5$. Real-space correlations $(\mathrm{c}, \mathrm{f})$ are presented in a square-root scale for better visibility.

approximation, is $\hat{H}_{n}=\hat{H}_{n}^{\text {lin }}+\hat{H}_{n}^{\text {local }}+\hat{H}_{n}^{\text {nonlocal }}$. Under these conditions in realistic WGAs only one combination of modes will contribute to the output quantum state $[19,23]$, hence we will just consider one mode for each component. Practically, different mode combinations can be chosen by using suitable pump and filtering specific signal and idler wavelengths.

The first part $\hat{H}_{n}^{\text {lin }}$ corresponds to the linear propagation of a photon pair in a waveguide array:

$$
\begin{aligned}
\hat{H}_{n}^{\operatorname{lin}} & =\hbar \sum_{n_{\mathrm{s}}}\left[\beta_{\mathrm{s}} \hat{a}_{\mathrm{s}}^{\dagger}\left(n_{\mathrm{s}}\right) \hat{a}_{\mathrm{s}}\left(n_{\mathrm{s}}\right)+c_{\mathrm{s}} \hat{a}_{\mathrm{s}}^{\dagger}\left(n_{\mathrm{s}}-1\right) \hat{a}_{\mathrm{s}}\left(n_{\mathrm{s}}\right)\right. \\
& \left.+c_{\mathrm{s}} \hat{a}_{\mathrm{s}}^{\dagger}\left(n_{\mathrm{s}}+1\right) \hat{a}_{\mathrm{s}}\left(n_{\mathrm{s}}\right)\right]+\hbar \sum_{n_{\mathrm{i}}}\left[\beta_{\mathrm{i}} \hat{a}_{\mathrm{i}}^{\dagger}\left(n_{\mathrm{i}}\right) \hat{a}_{\mathrm{i}}\left(n_{\mathrm{i}}\right)\right. \\
& \left.+c_{\mathrm{i}} \hat{a}_{\mathrm{i}}^{\dagger}\left(n_{\mathrm{i}}-1\right) \hat{a}_{\mathrm{i}}\left(n_{\mathrm{i}}\right)+c_{\mathrm{i}} \hat{a}_{\mathrm{i}}^{\dagger}\left(n_{\mathrm{i}}+1\right) \hat{a}_{\mathrm{i}}\left(n_{\mathrm{i}}\right)\right]+ \text { H.c.. }
\end{aligned}
$$

where $\beta_{m}$ are the propagation constants, $\hat{a}_{m}^{\dagger}\left(\hat{a}_{m}\right)$ are the creation (destruction) operators for the contributing set of modes, and $n$ is the waveguide number.

The second part $\hat{H}_{n}^{\text {local }}$ is responsible for the local nonlinear interaction between signal, idler and pump photons, where all three photons are in the same waveguide:

$$
\hat{H}_{n}^{\text {local }}=\mathrm{i} \hbar \gamma_{l} \sum_{n_{\mathrm{p}}} \hat{a}_{\mathrm{s}}^{\dagger}\left(n_{\mathrm{p}}\right) \hat{a}_{\mathrm{i}}^{\dagger}\left(n_{\mathrm{p}}\right) a_{\mathrm{p}}\left(n_{\mathrm{p}}\right)+\text { H.c.. }
$$

Here $a_{\mathrm{p}}$ is the classical pump amplitude across the waveguides.

The third part $\hat{H}_{n}^{\text {nonlocal }}$ corresponds to nonlocal nonlinear interactions, allowing a pump photon to split into a pair of signal 

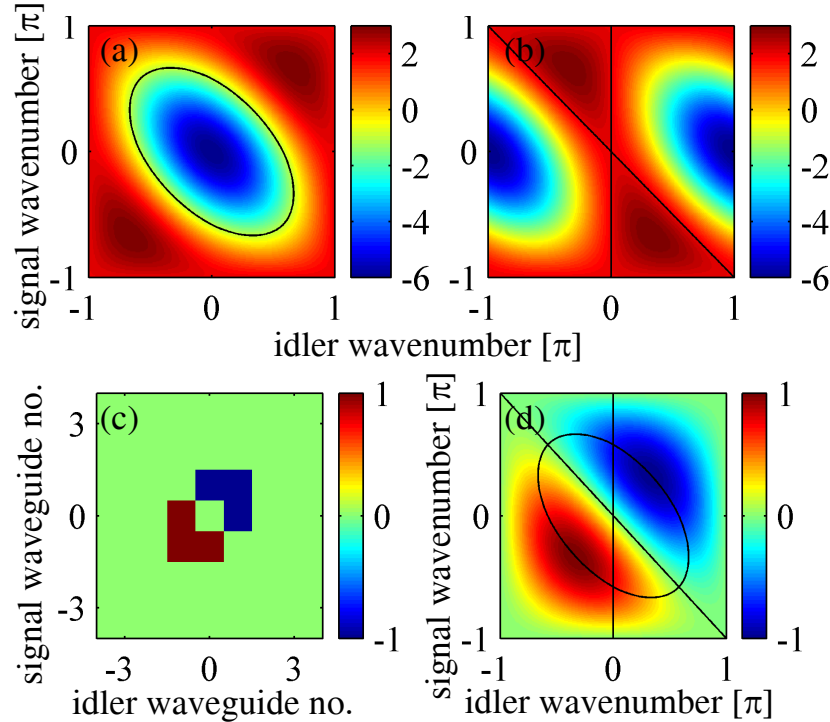

Fig. 3. SPDC with nonlocal nonlinear interaction. Phase mismatch functions with (a) $S_{\mathrm{p}}=-1, S_{\mathrm{s}}=1, S_{\mathrm{i}}=1$ and (b) $S_{\mathrm{p}}=1, S_{\mathrm{s}}=-1, S_{\mathrm{i}}=1$. The isolines mark the regions of phase-matching for (a) $\Delta \beta_{0}=1$ and (b) $\Delta \beta_{0}=-2$. (c) Possible biphotons for pump photon in waveguide 0 and nonlocal nonlinearity and (d) corresponding effective nonlinear coefficient. The isolines mark the same wavenumber regions as in $(a, b)$.

and idler photons, while at least one of the photons is located in a different waveguide:

$$
\begin{aligned}
& \hat{H}_{n}^{\text {nonlocal }}=\mathrm{i} \hbar \gamma_{\mathrm{s}} \sum_{n_{\mathrm{p}}} \hat{a}_{\mathrm{i}}^{\dagger}\left(n_{\mathrm{p}}\right) a_{\mathrm{p}}\left(n_{\mathrm{p}}\right)\left[S \hat{a}_{\mathrm{s}}^{\dagger}\left(n_{\mathrm{p}}-1\right)+\hat{a}_{\mathrm{s}}^{\dagger}\left(n_{\mathrm{p}}+1\right)\right] \\
& +\mathrm{i} \hbar \gamma_{\mathrm{i}} \sum_{n_{\mathrm{p}}} \hat{a}_{\mathrm{s}}^{\dagger}\left(n_{\mathrm{p}}\right) a_{\mathrm{p}}\left(n_{\mathrm{p}}\right)\left[S \hat{a}_{\mathrm{i}}^{\dagger}\left(n_{\mathrm{p}}-1\right)+\hat{a}_{\mathrm{i}}^{\dagger}\left(n_{\mathrm{p}}+1\right)\right] \\
& +\mathrm{i} \hbar \gamma_{\mathrm{p}} \sum_{n_{\mathrm{p}}} \hat{a}_{\mathrm{s}}^{\dagger}\left(n_{\mathrm{p}}\right) \hat{a}_{\mathrm{i}}^{\dagger}\left(n_{\mathrm{p}}\right)\left[S a_{\mathrm{p}}\left(n_{\mathrm{p}}-1\right)+a_{\mathrm{p}}\left(n_{\mathrm{p}}+1\right)\right]+\text { H.c. }
\end{aligned}
$$

This Hamiltonian can be converted from real space to wavevector- or $k$-space via a Fourier transform [13, 28]. In the rest of the paper we consider the regime of weak pump and focus on the generation of photon pairs only. The evolution of the system in the moving frame is described by the Schrödinger equation $i \hbar V \partial|\Psi(z)\rangle / \partial z \approx \hat{H}_{k}[|\Psi(z)\rangle+|0\rangle]$ with the photon-pair wavefunction $|\Psi(z)\rangle=\iint \mathrm{d} k_{\mathrm{s}} \mathrm{d} k_{\mathrm{i}} \Phi\left(k_{\mathrm{s}}, k_{\mathrm{i}}, z\right) \hat{a}_{\mathrm{s}}^{\dagger}\left(k_{\mathrm{s}}\right) \hat{a}_{\mathrm{i}}^{\dagger}\left(k_{\mathrm{i}}\right)|0\rangle$, where $V$ is the phase velocity. The joint-spectral density $\Phi\left(k_{\mathrm{s}}, k_{\mathrm{i}}, z\right)$ after a propagation length $L$ is [14]:

$$
\begin{aligned}
& \Phi\left(k_{\mathrm{s}}, k_{\mathrm{i}}, L\right)=L \tilde{\gamma}\left(k_{\mathrm{s}}, k_{\mathrm{i}}\right) \tilde{a}_{\mathrm{p}}\left(k_{\mathrm{s}}+k_{\mathrm{i}}\right) \operatorname{sinc}\left[\frac{L}{2}\left(\Delta \beta_{0}+\Delta \beta_{\text {array }}\right)\right] \\
& \exp \left[-i L\left(\frac{\Delta \beta_{0}}{2}+\frac{\Delta \beta_{\text {array }}}{2}+\beta_{\mathrm{p}}+2 c_{\mathrm{p}} \cos \left(k_{\mathrm{s}}+k_{\mathrm{i}}\right)\right)\right],
\end{aligned}
$$

where the phase mismatch of a single waveguide is $\Delta \beta_{0}=$ $\beta_{\mathrm{s}}+\beta_{\mathrm{i}}-\beta_{\mathrm{p}}$, and the additional phase-mismatch introduced by coupling in the WGA is $\Delta \beta_{\text {array }}=2 c_{\mathrm{s}} \cos \left(k_{\mathrm{s}}\right)+2 c_{\mathrm{i}} \cos \left(k_{\mathrm{i}}\right)-$ $2 c_{\mathrm{p}} \cos \left(k_{\mathrm{s}}+k_{\mathrm{i}}\right)$. Here $k_{\mathrm{s}}, k_{\mathrm{i}}$ are the transverse components of the wave-vectors for signal and idler photons and $\tilde{a}_{\mathrm{p}}, \tilde{\gamma}$ are the pump and nonlinearity profiles in $k$-space, respectively. Below $\tilde{a}_{\mathrm{p}}\left(k_{\mathrm{s}}+k_{\mathrm{i}}\right)$ will be considered constant, corresponding
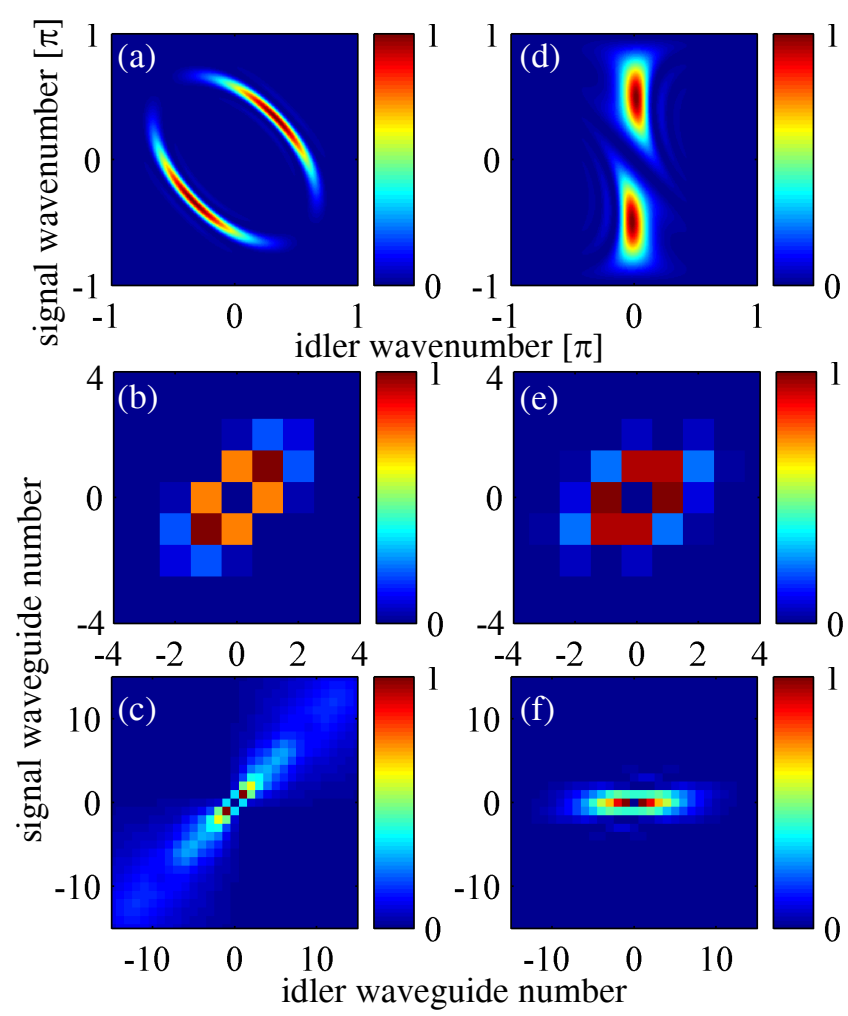

Fig. 4. SPDC with nonlocal nonlinear interaction and (a-c) $S_{\mathrm{p}}=-1, S_{\mathrm{s}}=1, S_{\mathrm{i}}=1, \Delta \beta_{0}=1$ and (d-f) $S_{\mathrm{p}}=1, S_{\mathrm{s}}=-1$, $S_{\mathrm{i}}=1, \Delta \beta_{0}=-2$. Correlations in $(\mathrm{a}, \mathrm{d}) k$-space for propagation length $L=5$ and in $(\mathrm{b}, \mathrm{c}, \mathrm{e}, \mathrm{f})$ real space for propagation lengths (b,e) $L=0.5$, and $(\mathrm{c}, \mathrm{f}) L=5$, respectively.

to pumping a single waveguide. Biphoton correlations are $\Gamma\left(k_{\mathrm{s}}, k_{\mathrm{i}}\right)=\left|\Phi\left(k_{\mathrm{s}}, k_{\mathrm{i}}, L\right)\right|^{2}$ in $k$-space or $\Gamma\left(n_{\mathrm{s}}, n_{\mathrm{i}}\right)=(2 \pi)^{-2}$ $\left|\iint \mathrm{d} k_{\mathrm{s}} \mathrm{d} k_{\mathrm{i}} \exp \left(i k_{\mathrm{s}} n_{\mathrm{s}}\right) \exp \left(i k_{\mathrm{i}} n_{\mathrm{i}}\right) \Phi\left(k_{\mathrm{s}}, k_{\mathrm{i}}, L\right)\right|^{2}$ in real space.

We now compare photon pair generation with local and nonlocal SPDC. To this end we will use quantities normalized to the idler coupling constant $c_{\mathrm{i}}$. We assume the magnitude of the normalized coupling constants of all modes equal, $\left|c_{m}\right|=1$ [23] and furthermore set all nonlocal nonlinear coefficients equal. However, our theoretical formulations are valid for all normalized coupling values. In Fig. 2 we show the phase mismatch and correlations for a case with $S=1$ and local nonlinearity, where the nonlinear interaction coefficient in $k$-space is $\tilde{\gamma}\left(k_{\mathrm{s}}, k_{\mathrm{i}}\right)=\gamma_{l}$. We consider all modes even, however, other mode combinations with $S=1$ would result in the same correlations. For a small negative single-waveguide phase mismatch $\Delta \beta_{0}=-1$ the phase-matching curve in $k$-space is an ellipse [Fig. 2(a)], leading to the generation of elliptical $k$-space correlations between signal and idler photons [Fig. 2(b)]. Real space correlations in this case are not very pronounced [Fig. 2(c)]. When the single-waveguide phase mismatch is changed to $\Delta \beta_{0}=2$, the phase-matching curve [Fig. 2(d)] and the corresponding $k$-space two-photon correlations become cross-shaped, which in response leads to 6-petal real space biphoton correlations [Fig. 2(e)].

For nonlocal nonlinearity we consider two cases with parameters similar to previously discussed local cases, $S_{\mathrm{p}}=-1, S_{\mathrm{s}}=1$, $S_{\mathrm{i}}=1, \Delta \beta_{0}=1$ and $S_{\mathrm{p}}=1, S_{\mathrm{s}}=-1, S_{\mathrm{i}}=1, \Delta \beta_{0}=-2$. Note that the signs of the phase mismatch functions are reversed with respect to the cases with local nonlinearity, however, the shapes 
of the phase-matching curves remain an ellipse [Fig. 3(a)] and a cross [Fig. 3(b)], similar to the local nonlinear regime, but with inverse signs of single-waveguide phase mismatch.

Importantly, in the nonlocal regime with $S=-1$ the nonlinear interactions are qualitatively different from the local case. In real space, possible locations for the generation of signal and idler photons are shifted from the pump waveguide and also provide different phases depending on the direction of the shift as shown in Fig. 3(c). This results in an inhomogeneous nonlinear coefficient in $k$-space [26], $\tilde{\gamma}\left(k_{\mathrm{s}}, k_{\mathrm{i}}\right)=$ $2 i \sin \left(k_{\mathrm{s}}\right)\left[\gamma_{\mathrm{s}}-\gamma_{\mathrm{p}} \cos \left(k_{\mathrm{s}}\right)\right]+2 i \sin \left(k_{\mathrm{i}}\right)\left[\gamma_{\mathrm{i}}-\gamma_{\mathrm{p}} \cos \left(k_{\mathrm{i}}\right)\right]$. The imaginary part of $\tilde{\gamma}\left(k_{\mathrm{s}}, k_{\mathrm{i}}\right)$ is plotted in Fig. 3(d) together with the lines of phase matching from Fig. 3(a,b). As the overlap between the nonlinearity and the phase matching curves determines the correlations, these are strongly influenced by such an anisotropic nonlinearity. Different spectral signal and idler components are generated with different efficiency. Most notably, anti-bunching in $k$-space with $k_{\mathrm{s}}=-k_{\mathrm{i}}$ is suppressed, independent of the phase mismatch. Furthermore, quantum states generated by this anisotropic nonlinearity will be entangled, independent of the propagation length. This is evident from the nonlinear coefficient given above, which consists of two products of signal and idler dependent functions. Hence, the Schmidt number of the generated two-photon state is at least 2 , indicating entanglement, which is in contrast to photon-pair generation in WGAs with local nonlinearity, where the Schmidt number for short lengths is 1 [19] and entangled states are only generated for larger propagation lengths.

In the case of positive single-waveguide phase mismatch $\Delta \beta_{0}=1$, the $k$-space correlations in the nonlocal regime show an interrupted ellipse [Fig. 4(a)]. The corresponding real-space correlations show very pronounced spatial bunching, that grows stronger as the length of the WGA is increased [Fig. 4(b-d)]. Such clear spatial bunching does not appear in homogeneous WGAs with local nonlinear interactions [13]. For negative singlewaveguide phase mismatch $\Delta \beta_{0}=-2$ the correlations change dramatically. While the magnitudes of the normalized coupling coefficients for all interacting waves are equal to one, the $k$-space correlations are narrow for the idler photon and elongated for the signal photon [Fig. 4(e)]. For small and medium $L$ [Fig. 4(f,g)] the real-space correlations resemble the generation pattern from Fig. 3(c). For larger $L$, the idler spreads across the WGA due to linear coupling whereas the signal coupling is suppressed [Fig. 4(h)]. This effect could be used to herald an idler photon generated in a $\mathrm{W}$ state across many waveguides by detecting a signal photon in a central waveguide. This demonstrates that nonlocal nonlinearities are promising as additional tool to control biphoton spatial correlations, beyond utilizing waveguide coupling and phase matching. Furthermore, it might be interesting to study nonlocal biphoton generation in other systems with effective nonlocal response, e.g. metamaterials [29].

To conclude, we have proposed an integrated photonic system where locality for photon-pair generation based on SPDC can be violated, leading to the annihilation of a pump photon in one waveguide, and creation of a pair of photons in other waveguides. This phenomenon opens new opportunities for the design of on-chip photon-pair sources.

Funding. Australian Research Council (Discovery Projects DP130100135 and DP160100619), Erasmus Mundus NANOPHI (contract 2013 5659/002- 001), Alexander von Humboldt Foundation, Australia-Germany Joint Research Co-operation Scheme of Universities Australia, German Academic Exchange Service, Federal Ministry of Education and Research (FKZ 03ZZ0434).

\section{REFERENCES}

1. D. C. Burnham and D. L. Weinberg, Phys. Rev. Lett. 25, 84 (1970).

2. J. P. Dowling, Schrödinger's killer app: race to build the world's first quantum computer (CRC Press, 2013).

3. P. G. Kwiat, K. Mattle, H. Weinfurter, A. Zeilinger, A. V. Sergienko, and Y. Shih, Phys. Rev. Lett. 75, 4337 (1995).

4. Z. H. Levine, J. Fan, J. Chen, and A. L. Migdall, Opt. Express 19, 6724 (2011).

5. M. V. Fedorov, M. A. Efremov, P. A. Volkov, E. V. Moreva, S. S. Straupe, and S. P. Kulik, Phys. Rev. Lett. 99, 063901 (2007).

6. A. Christ, K. Laiho, A. Eckstein, K. N. Cassemiro, and C. Silberhorn, New Journal of Physics 13, 033027 (2011).

7. S. Tanzilli, A. Martin, F. Kaiser, M. De Micheli, O. Alibart, and D. Ostrowsky, Laser \& Photonics Reviews 6, 115 (2012).

8. H. Jin, F. M. Liu, P. Xu, J. L. Xia, M. L. Zhong, Y. Yuan, J. W. Zhou, Y. X. Gong, W. Wang, and S. N. Zhu, Phys. Rev. Lett. 113, 103601 (2014).

9. W. Silverstone, D. Bonneau, K. Ohira, N. Suzuki, H. Yoshida, N. lizuka, M. Ezaki, M. C. Natarajan, G. Tanner, H. R. Hadfield, V. Zwiller, D. G. Marshall, G. J. Rarity, L. J. O'Brien, and G. M. Thompson, Nat Photon 8, 104 (2014).

10. R. Kruse, L. Sansoni, S. Brauner, R. Ricken, C. S. Hamilton, I. Jex, and C. Silberhorn, Physical Review A 92, 053841 (2015).

11. F. Setzpfandt, A. S. Solntsev, J. Titchener, C. W. Wu, C. L. Xiong, R. Schiek, T. Pertsch, D. N. Neshev, and A. A. Sukhorukov, Laser \& Photonics Reviews 10, 131 (2016).

12. A. S. Solntsev, A. A. Sukhorukov, D. N. Neshev, and Y. S. Kivshar, Phys. Rev. Lett. 108, 023601 (2012).

13. A. S. Solntsev, A. A. Sukhorukov, D. N. Neshev, and Y. S. Kivshar, Opt. Express 20, 27441 (2012).

14. R. Kruse, F. Katzschmann, A. Christ, A. Schreiber, S. Wilhelm, K. Laiho, A. Gábris, C. S. Hamilton, I. Jex, and C. Silberhorn, New Journal of Physics 15, 083046 (2013).

15. D. A. Antonosyan, A. S. Solntsev, and A. A. Sukhorukov, Phys. Rev. A 90, 043845 (2014).

16. C. S. Hamilton, R. Kruse, L. Sansoni, C. Silberhorn, and I. Jex, Phys. Rev. Lett. 113, 083602 (2014).

17. J. G. Titchener, A. S. Solntsev, and A. A. Sukhorukov, Phys. Rev. A 92 , 033819 (2015).

18. D. Leykam, A. S. Solntsev, A. A. Sukhorukov, and A. S. Desyatnikov, Phys. Rev. A 92, 033815 (2015).

19. A. S. Solntsev, F. Setzpfandt, A. S. Clark, C. W. Wu, M. J. Collins, C. Xiong, A. Schreiber, F. Katzschmann, F. Eilenberger, R. Schiek, W. Sohler, A. Mitchell, C. Silberhorn, B. J. Eggleton, T. Pertsch, A. A. Sukhorukov, D. N. Neshev, and Y. S. Kivshar, Phys. Rev. X 4, 031007 (2014).

20. D. Strekalov, A. B. Matsko, A. A. Savchenkov, and L. Maleki, Phys. Rev. A 71, 041803 (2005).

21. L. Zhang, A. B. U'ren, R. Erdmann, K. A. O???Donnell, C. Silberhorn, K. Banaszek, and I. A. Walmsley, Journal of Modern Optics 54, 707 (2007).

22. K. Y. Spasibko, D. A. Kopylov, T. V. Murzina, G. Leuchs, and M. V. Chekhova, Opt. Lett. 41, 2827 (2016).

23. F. Setzpfandt, M. Falkner, T. Pertsch, W. Sohler, and R. Schiek, Applied Physics Letters 102, 081104 (2013).

24. F. Setzpfandt, A. A. Sukhorukov, D. N. Neshev, R. Schiek, Y. S. Kivshar, and T. Pertsch, Phys. Rev. Lett. 105, 233905 (2010).

25. F. Setzpfandt, A. A. Sukhorukov, D. N. Neshev, R. Schiek, A. S. Solntsev, R. Ricken, Y. Min, W. Sohler, Y. S. Kivshar, and T. Pertsch, Opt. Express 19, 23188 (2011).

26. F. Setzpfandt, W. Sohler, R. Schiek, and T. Pertsch, Phys. Rev. A 92 , 043832 (2015).

27. A. Yariv and P. Yeh, Optical waves in crystals, vol. 10 (Wiley, New York, 1984).

28. M. Grafe, A. S. Solntsev, R. Keil, A. A. Sukhorukov, M. Heinrich, A. Tunnermann, S. Nolte, A. Szameit, and Y. S. Kivshar, Scientific Reports 2, 562 (2012).

29. A. N. Poddubny, I. V. lorsh, and A. A. Sukhorukov, Phys. Rev. Lett. 117, 123901 (2016). 


\section{FULL REFERENCES}

1. D. C. Burnham and D. L. Weinberg, "Observation of simultaneity in parametric production of optical photon pairs," Phys. Rev. Lett. 25, 84-87 (1970)

2. J. P. Dowling, Schrödinger's killer app: race to build the world's first quantum computer (CRC Press, 2013).

3. P. G. Kwiat, K. Mattle, H. Weinfurter, A. Zeilinger, A. V. Sergienko, and Y. Shih, "New high-intensity source of polarization-entangled photon pairs," Phys. Rev. Lett. 75, 4337-4341 (1995).

4. Z. H. Levine, J. Fan, J. Chen, and A. L. Migdall, "Polarization-entangled photon pairs from a periodically poled crystalline waveguide," Opt. Express 19, 6724-6740 (2011).

5. M. V. Fedorov, M. A. Efremov, P. A. Volkov, E. V. Moreva, S. S. Straupe, and S. P. Kulik, "Anisotropically and high entanglement of biphoton states generated in spontaneous parametric down-conversion," Phys. Rev. Lett. 99, 063901 (2007).

6. A. Christ, K. Laiho, A. Eckstein, K. N. Cassemiro, and C. Silberhorn, "Probing multimode squeezing with correlation functions," New Journal of Physics 13, 033027 (2011).

7. S. Tanzilli, A. Martin, F. Kaiser, M. De Micheli, O. Alibart, and D. Ostrowsky, "On the genesis and evolution of integrated quantum optics," Laser \& Photonics Reviews 6, 115-143 (2012).

8. H. Jin, F. M. Liu, P. Xu, J. L. Xia, M. L. Zhong, Y. Yuan, J. W. Zhou, Y. X. Gong, W. Wang, and S. N. Zhu, "On-chip generation and manipulation of entangled photons based on reconfigurable lithium-niobate waveguide circuits," Phys. Rev. Lett. 113, 103601 (2014).

9. W. Silverstone, D. Bonneau, K. Ohira, N. Suzuki, H. Yoshida, N. lizuka, M. Ezaki, M. C. Natarajan, G. Tanner, H. R. Hadfield, V. Zwiller, D. G. Marshall, G. J. Rarity, L. J. O'Brien, and G. M. Thompson, "On-chip quantum interference between silicon photon-pair sources," Nat Photon 8, 104-108 (2014).

10. R. Kruse, L. Sansoni, S. Brauner, R. Ricken, C. S. Hamilton, I. Jex, and C. Silberhorn, "Dual-path source engineering in integrated quantum optics," Physical Review A 92, 053841 (2015).

11. F. Setzpfandt, A. S. Solntsev, J. Titchener, C. W. Wu, C. L. Xiong, R. Schiek, T. Pertsch, D. N. Neshev, and A. A. Sukhorukov, "Tunable generation of entangled photons in a nonlinear directional coupler," Laser \& Photonics Reviews 10, 131-136 (2016).

12. A. S. Solntsev, A. A. Sukhorukov, D. N. Neshev, and Y. S. Kivshar, "Spontaneous parametric down-conversion and quantum walks in arrays of quadratic nonlinear waveguides," Phys. Rev. Lett. 108, 023601 (2012).

13. A. S. Solntsev, A. A. Sukhorukov, D. N. Neshev, and Y. S. Kivshar, "Photon-pair generation in arrays of cubic nonlinear waveguides," Opt. Express 20, 27441-27446 (2012).

14. R. Kruse, F. Katzschmann, A. Christ, A. Schreiber, S. Wilhelm, K. Laiho, A. Gábris, C. S. Hamilton, I. Jex, and C. Silberhorn, "Spatio-spectral characteristics of parametric down-conversion in waveguide arrays," New Journal of Physics 15, 083046 (2013).

15. D. A. Antonosyan, A. S. Solntsev, and A. A. Sukhorukov, "Effect of loss on photon-pair generation in nonlinear waveguide arrays," Phys. Rev. A 90, 043845-10 (2014).

16. C. S. Hamilton, R. Kruse, L. Sansoni, C. Silberhorn, and I. Jex, "Driven quantum walks," Phys. Rev. Lett. 113, 083602 (2014).

17. J. G. Titchener, A. S. Solntsev, and A. A. Sukhorukov, "Generation of photons with all-optically-reconfigurable entanglement in integrated nonlinear waveguides," Phys. Rev. A 92, 033819-12 (2015).

18. D. Leykam, A. S. Solntsev, A. A. Sukhorukov, and A. S. Desyatnikov, "Lattice topology and spontaneous parametric down-conversion in quadratic nonlinear waveguide arrays," Phys. Rev. A 92, 033815 (2015).

19. A. S. Solntsev, F. Setzpfandt, A. S. Clark, C. W. Wu, M. J. Collins, C. Xiong, A. Schreiber, F. Katzschmann, F. Eilenberger, R. Schiek, W. Sohler, A. Mitchell, C. Silberhorn, B. J. Eggleton, T. Pertsch, A. A. Sukhorukov, D. N. Neshev, and Y. S. Kivshar, "Generation of nonclassical biphoton states through cascaded quantum walks on a nonlinear chip," Phys. Rev. X 4, 031007 (2014).

20. D. Strekalov, A. B. Matsko, A. A. Savchenkov, and L. Maleki, "Relationship between quantum two-photon correlation and classical spectrum of light," Phys. Rev. A 71, 041803 (2005).

21. L. Zhang, A. B. U'ren, R. Erdmann, K. A. O???Donnell, C. Silberhorn, K. Banaszek, and I. A. Walmsley, "Generation of highly entangled photon pairs for continuous variable bell inequality violation," Journal of Modern Optics 54, 707-719 (2007).

22. K. Y. Spasibko, D. A. Kopylov, T. V. Murzina, G. Leuchs, and M. V. Chekhova, "Ring-shaped spectra of parametric downconversion and entangled photons that never meet," Opt. Lett. 41, 2827-2830 (2016)

23. F. Setzpfandt, M. Falkner, T. Pertsch, W. Sohler, and R. Schiek, "Bandstructure measurement in nonlinear optical waveguide arrays," Applied Physics Letters 102, 081104 (2013).

24. F. Setzpfandt, A. A. Sukhorukov, D. N. Neshev, R. Schiek, Y. S. Kivshar, and T. Pertsch, "Phase transitions of nonlinear waves in quadratic waveguide arrays," Phys. Rev. Lett. 105, 233905 (2010).

25. F. Setzpfandt, A. A. Sukhorukov, D. N. Neshev, R. Schiek, A. S. Solntsev, R. Ricken, Y. Min, W. Sohler, Y. S. Kivshar, and T. Pertsch, "Spectral pulse transformations and phase transitions in quadratic nonlinear waveguide arrays," Opt. Express 19, 23188-23201 (2011).

26. F. Setzpfandt, W. Sohler, R. Schiek, and T. Pertsch, "Nonlinear coupling in discrete optical waveguide arrays with quadratic nonlinearity," Phys. Rev. A 92, 043832 (2015).

27. A. Yariv and P. Yeh, Optical waves in crystals, vol. 10 (Wiley, New York, 1984).

28. M. Grafe, A. S. Solntsev, R. Keil, A. A. Sukhorukov, M. Heinrich, A. Tunnermann, S. Nolte, A. Szameit, and Y. S. Kivshar, "Biphoton generation in quadratic waveguide arrays: A classical optical simulation," Scientific Reports 2, 562-5 (2012).

29. A. N. Poddubny, I. V. lorsh, and A. A. Sukhorukov, "Generation of photon-plasmon quantum states in nonlinear hyperbolic metamaterials," Phys. Rev. Lett. 117, 123901 (2016). 